\title{
SENTINEL1: Two-Season Study of Respiratory Syncytial Virus Hospitalizations among U.S. Infants Born at 29 to 35 Weeks' Gestational Age Not Receiving Immunoprophylaxis
}

Evan J. Anderson, $M^{1}$ John P. DeVincenzo, $M^{2}$ Eric A. F. Simões, MBBS, DCH, $\mathrm{MD}^{3}$

Leonard R. Krilov, MD ${ }^{4}$ Michael L. Forbes, MD ${ }^{5}$ Pia S. Pannaraj, MD, MPH ${ }^{6}$

Claudia M. Espinosa, MD, MSc ${ }^{7}$ Robert C. Welliver, MD ${ }^{8}$ Leslie I. Wolkoff, MD ${ }^{9}$ Ram Yogev, MD ${ }^{10}$

Paul A. Checchia, MD ${ }^{11}$ Joseph B. Domachowske, MD ${ }^{12}$ Natasha Halasa, MD, MPH ${ }^{13}$

Scott J. McBride, MA ${ }^{14}$ Veena R. Kumar, MD, MPH ${ }^{15}$ Kimmie K. McLaurin, MS ${ }^{15}$

Christopher P. Rizzo, MD ${ }^{15}$ Christopher S. Ambrose, MD, MBA ${ }^{15}$

${ }^{1}$ Departments of Pediatrics and Medicine, Emory University School of Medicine, Atlanta, Georgia

2 Departments of Pediatrics and Microbiology, Immunology, and Biochemistry, University of Tennessee School of Medicine, Memphis, Tennessee

${ }^{3}$ Section of Pediatric Infectious Disease, University of Colorado School of Medicine, Colorado School of Public Health, Children's Hospital Colorado, Aurora, Colorado

${ }^{4}$ Division of Pediatric Infectious Disease, Children's Medical Center, NYU-Winthrop Hospital, Mineola, New York

5 Pediatric Critical Care Medicine, Akron Children's Hospital, Akron, Ohio

6 Division of Infectious Diseases, Children's Hospital Los Angeles, Los Angeles, California, Keck School of Medicine, University of Southern California, Los Angeles, California

${ }^{7}$ Pediatric Infectious Diseases, University of Louisville, Louisville, Kentucky

8 Pediatric Infectious Diseases, Children's Hospital at OU Medical

Center, Oklahoma City, Oklahoma
Address for correspondence Christopher P. Rizzo, MD, Sobi Inc., 890 Winter Street, Suite 200, Waltham, MA 02451

(e-mail: christopher.rizzo@sobi.com).

${ }^{9}$ Division of Neonatology, Connecticut Children's Medical Center, Hartford, Connecticut

10 Department of Pediatrics, Ann \& Robert H. Lurie Children's Hospital of Chicago, Feinberg School of Medicine, Northwestern University, Chicago, Illinois

${ }^{11}$ Cardiac Intensive Care, Texas Children's Hospital, Houston, Texas

12 Pediatrics: Infectious Disease, SUNY Upstate Medical University, Syracuse, New York

13 Pediatric Infectious Diseases, Vanderbilt University Medical Center, Nashville, Tennessee

${ }^{14}$ United BioSource Corporation, Ann Arbor, Michigan

${ }^{15}$ AstraZeneca, Gaithersburg, Maryland Am J Perinatol 2020;37:421-429.

\section{Abstract \\ Keywords \\ - respiratory syncytial virus \\ - infants \\ - palivizumab \\ - immunoprophylaxis \\ - hospitalizations}

Objective The SENTINEL1 observational study characterized confirmed respiratory syncytial virus hospitalizations (RSVH) among U.S. preterm infants born at 29 to 35 weeks' gestational age (WGA) not receiving respiratory syncytial virus (RSV) immunoprophylaxis (IP) during the 2014 to 2015 and 2015 to 2016 RSV seasons.

Study Design All laboratory-confirmed RSVH at participating sites during the 2014 to 2015 and 2015 to 2016 RSV seasons (October 1-April 30) lasting $\geq 24$ hours among preterm infants 29 to 35 WGA and aged $<12$ months who did not receive RSV IP within 35 days before onset of symptoms were identified and characterized.

Results Results were similar across the two seasons. Among infants with communityacquired RSVH ( $N=1,378$ ), $45 \%$ were admitted to the intensive care unit (ICU) and $19 \%$ required invasive mechanical ventilation (IMV). There were two deaths. Infants aged $<6$ months accounted for $78 \%$ of RSVH observed, $84 \%$ of ICU admissions, and $91 \%$ requiring IMV. Among infants who were discharged from their birth hospitalization during the RSV season, $82 \%$ of RSVH occurred within 60 days of birth hospitalization discharge.

Conclusion Among U.S. preterm infants 29 to 35 wGA not receiving RSV IP, RSVH are often severe with almost one-half requiring ICU admission and about one in five needing IMV. received

December 27, 2018

accepted

January 22, 2019

published online

April 16, 2019
Copyright $\odot 2020$ by Thieme Medical

DOI https://doi.org/

10.1055/s-0039-1681014. ISSN 0735-1631.
Publishers, Inc., 333 Seventh Avenue, New York, NY 10001, USA. Tel: +1(212) 760-0888.
License terms

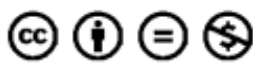


Respiratory syncytial virus (RSV) is the most common cause of hospitalization due to lower respiratory tract infection in infants and young children. ${ }^{1}$ RSV is estimated to cause up to $75 \%$ of all infant bronchiolitis and $40 \%$ of all pediatric pneumonias. ${ }^{2,3}$ Preterm ( $\leq 35$ weeks' gestational age [wGA]) infants and young children with certain underlying cardiac or pulmonary conditions are at high risk of serious sequelae from RSV infection. ${ }^{4-6}$ Infants who are born preterm represent the largest population of high-risk children. Studies conducted prior to the widespread use of palivizumab showed that preterm infants had at least twice the risk of RSV hospitalization (RSVH) and were more likely to require intensive care unit (ICU) admission and mechanical ventilation compared with full-term infants. ${ }^{4,7,8}$

Considerable efforts have been made to develop RSV vaccines for prevention and RSV antivirals for treatment, but the U.S. Food and Drug Administration (FDA) approval of such prevention and treatment strategies remains years away. ${ }^{9-11}$ RSV immunoprophylaxis (IP) with palivizumab remains the only FDA-approved intervention for the prevention of serious RSV disease. Based on randomized, placebocontrolled studies, palivizumab is indicated to prevent serious lower respiratory tract disease caused by RSV in preterm infants born at $\leq 35$ wGA and children $\leq 24$ months of age with chronic lung disease of prematurity (CLDP) or hemodynamically significant congenital heart disease (HS-CHD). In preterm infants without CLDP or HS-CHD, palivizumab reduced RSVH by 78 to $82 \%,{ }^{12,13}$ an observation confirmed in a test-negative, case-control effectiveness study. ${ }^{14}$

The American Academy of Pediatrics Committee on Infectious Diseases (COID) first published recommendations for the use of RSV IP shortly after its licensure in 1998 with subsequent updates and revisions. ${ }^{15-20}$ The COID guidance continues to recommend RSV IP for infants with CLDP and HSCHD. In 2014, the COID no longer recommended RSV IP for preterm infants born at 29 to 35 wGA except for those with another qualifying medical condition, such as CLDP or HS$\mathrm{CHD} .{ }^{20}$ The rationale for the change was that infants born at 29 to $35 \mathrm{wGA}$ had a rate of RSVH similar to term infants. ${ }^{20}$ The 2014 COID guidance has been controversial. ${ }^{21}$ The National Perinatal Association also issued guidelines in 2014; however, they recommended broader use of RSV IP than did the COID. ${ }^{22}$

The purpose of the two-season SENTINEL1 study was to characterize confirmed RSVH among U.S. preterm infants born at 29 to 35 wGA who had not received RSV IP. Results for the 2014 to 2015 RSV season have been reported. ${ }^{23}$ The current analysis provides results for an additional RSV season and the combined two-season cohorts (2014-2015 and 2015-2016), representing the largest and most detailed description of RSVconfirmed hospitalizations among U.S. preterm infants.

\section{Materials and Methods}

\section{Study Design}

The SENTINEL1 study design has been described previously. ${ }^{23}$ A total of 46 sites across the United States participated in the two-season observational study (ClinicalTrials. gov identifier: NCT02273882): Thirty-nine sites participated in both seasons; four sites participated in 2014 to 2015 (season 1) only; and three in 2015 to 2016 (season 2) only. The study eligibility period was predefined as October 1 through April 30 in each of the two seasons.

\section{Patient Population and Data Collection}

\section{Eligible Infants}

Infants were eligible if they were (1) born at 29 to 35 wGA (29 weeks, 0 days -35 weeks, 6 days), (2) hospitalized $\geq 24$ hours for laboratory-confirmed community-acquired or nosocomial RSV disease (index RSVH), and (3) aged $<12$ months at the time of the index RSVH. RSV diagnosis was confirmed through local standard-of-care clinical laboratory testing performed for the current respiratory illness. Infants were excluded if they had received RSV IP within 35 days before the onset of respiratory symptoms associated with the index RSVH. Infants meeting the eligibility requirements were systematically identified prospectively and/or retrospectively at each site. Deidentified descriptive characteristics were collected for all eligible infants, including the infant's gestational age, birth month, insurance status (public or private), hospital admission date, hospital length of stay (LOS), need for ICU admission, ICU LOS, need for invasive mechanical ventilation (IMV), and survival status at discharge. IMV included intubation for conventional mechanical ventilation, continuous positive airway pressure, extracorporeal membrane oxygenation, high-frequency oscillatory ventilation, and/or jet ventilation. All patient care decisions, including admission or transfer to the ICU and/or need for IMV, were made independently from the study by the clinical teams caring for the patient.

\section{Enrolled Infants}

In an effort to balance the cohort by gestational age group, parents/guardians of every infant 29 to 32 wGA and every other infant 33 to 35 wGA were approached to enroll for indepth characterization of the RSVH. After obtaining informed consent from a parent/guardian, detailed data related to the infant's RSVH were collected. Infants were eligible for enrollment during the index RSVH (prospective enrollment) or following hospital discharge (retrospective enrollment). Additional data were collected on enrolled infants including date of birth, details of the birth hospitalization, presence of epidemiological risk factors for an RSVH, details of the index RSV illness including any prior or subsequent medically attended encounters, and hospital charges for the index RSVH based on insurance claim forms or billing records. Given the studyspecific differential enrollment of infants 29 to 32 wGA and 33 to $35 \mathrm{wGA}$, descriptions of these populations were provided separately. The study was reviewed and approved by either a local or central (Copernicus Group, Cary, NC) Institutional Review Board for all participating sites.

\section{Statistical Analysis}

Descriptive analyses were performed to characterize infants with RSVH overall and by gestational age group, for each season and the combined seasons. Potential bias in the enrolled population due to parental willingness or ability 
to consent was assessed by comparing the enrolled cohort to the eligible cohort. Statistical comparisons between gestational age groups were conducted using the Wilcoxon's ranksum test for continuous variables (hospitalization LOS and ICU LOS) and the Pearson's chi-square test for categorical variables (ICU admission and need for IMV). Testing was two sided and conducted at the $5 \%$ level of significance, with no adjustment for multiple comparisons. Stepwise logistic regression was used to identify factors having an impact on the frequency of ICU admission among the eligible infant cohorts. Factors from individual univariate analyses with a $p$-value $<0.2$ were considered candidates for the stepwise analysis. Infants with missing data were excluded from the analysis. The final multivariate model identified via this process was also used to analyze the need for IMV. The distributions of community-acquired RSVH by time from birth hospitalization discharge, month of birth hospitalization discharge, and birth month were also evaluated.

\section{Study Site Characteristics}

Sites completed surveys regarding their standard of care for provision of RSV IP as well as their approach to diagnostic RSV testing for infants hospitalized with acute respiratory illness.

\section{Results}

\section{Eligible Infants}

Over the two seasons, 1,398 infants met eligibility criteria. Among these, 1,378 (99\%) had community-acquired RSV disease and 20 (1\%) had nosocomial RSV disease; only the community-acquired cases were analyzed further (-Fig. 1). A total of 441 (32\%) were born at 29 to 32 wGA, 571 (41\%) at 33 to 34 wGA, and 366 (27\%) at 35 wGA (-Table 1 ).

RSVH, ICU admissions, and need for IMV were similar across the two studied seasons. During the 2014 to 2015 RSV season, infants aged $<6$ months accounted for 78,87 , and $92 \%$ of RSVH, ICU admissions, and need for IMV, respectively; similarly, during the 2015 to 2016 season infants aged $<6$ months accounted for 78,81 , and $90 \%$ of RSVH, ICU admission, and need for IMV, respectively (-Supplementary Fig. S1 [available in the online version]). The chronological age distribution of these outcomes for the combined seasons is shown in - Fig. 2. RSVH outcomes (overall hospital LOS, ICU admission, ICU LOS, and need for IMV) by gestational age group and by chronological age group are shown in - Tables 1 and 2, respectively. During both RSV seasons, earlier gestational age combined with younger chronological age was

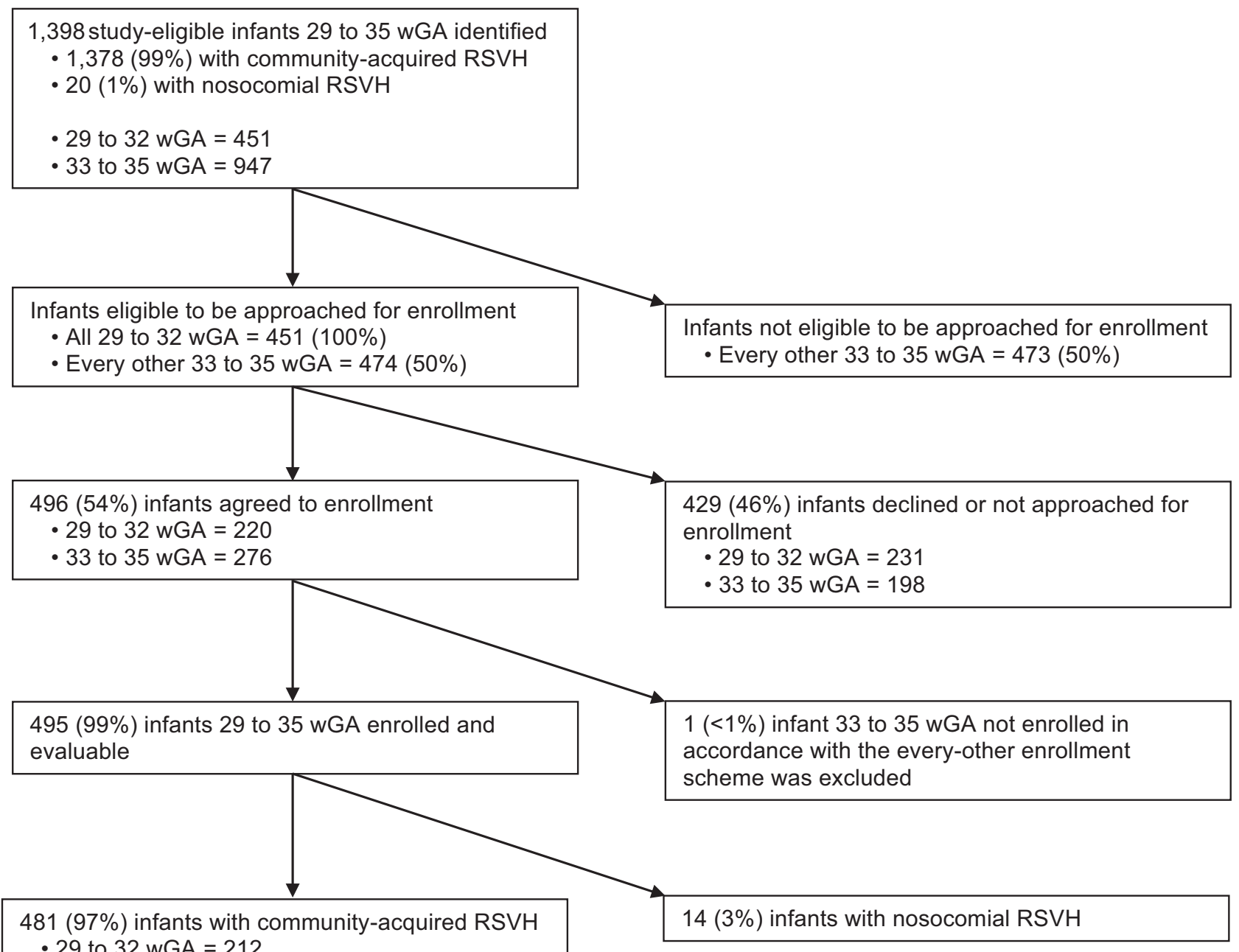

- 29 to 32 wGA $=212$

- 33 to 35 wGA $=269$

Fig. 1 Study population disposition. RSVH, respiratory syncytial virus hospitalization; wGA, weeks' gestational age. 
Table 1 Outcomes of community-acquired RSV hospitalizations among all study-eligible infants by gestational age group

\begin{tabular}{|c|c|c|c|c|c|c|c|c|c|}
\hline & \multicolumn{3}{|l|}{ 29-32 wGA } & \multicolumn{3}{|l|}{ 33-34 wGA } & \multicolumn{3}{|l|}{35 wGA } \\
\hline & $\begin{array}{l}2014-2015 \\
(n=237)\end{array}$ & $\begin{array}{l}2015-2016 \\
(n=204)\end{array}$ & $\begin{array}{l}\text { Combined } \\
\text { seasons } \\
(n=441)\end{array}$ & $\begin{array}{l}2014-2015 \\
(n=283)\end{array}$ & $\begin{array}{l}2015-2016 \\
(n=288)\end{array}$ & $\begin{array}{l}\text { Combined } \\
\text { seasons }^{\mathrm{a}} \\
(n=571)\end{array}$ & $\begin{array}{l}2014-2015 \\
(n=182)\end{array}$ & $\begin{array}{l}2015-2016 \\
(n=184)\end{array}$ & $\begin{array}{l}\text { Combined } \\
\text { seasons }^{\mathrm{a}} \\
(n=366)\end{array}$ \\
\hline \multicolumn{10}{|l|}{ Hospital LOS, days ${ }^{\mathrm{b}}$} \\
\hline Median (IQR) & $6(3-12)^{c}$ & $5(3-10)^{c}$ & $6(3-11)$ & $6(3-10)^{c}$ & $5(3-10)$ & $5(3-10)$ & $5(3-7)$ & $5(3-8)$ & $5(3-8)$ \\
\hline Mean (SD) & $10(10)$ & $10(16)$ & $10(13)$ & $9(12)$ & $8(10)$ & $9(11)$ & $7(11)$ & $8(11)$ & $7(11)$ \\
\hline Range & $1-67$ & $1-188$ & $1-188$ & $1-101$ & $1-85$ & $1-101$ & $1-135$ & $1-113$ & $1-135$ \\
\hline $\begin{array}{l}\text { ICU admission, } \\
n(\%)^{\mathrm{b}}\end{array}$ & $115(49)^{d}$ & $97(48)$ & $212(48)$ & $117(43)^{d}$ & $137(48)$ & $254(46)$ & $56(31)$ & $88(48)$ & $144(40)$ \\
\hline \multicolumn{10}{|l|}{ ICU LOS, days ${ }^{\mathrm{e}, \mathrm{f}}$} \\
\hline Median (IQR) & $8(3-14)$ & $6(3-11)$ & $7(3-13)$ & $6(3-12)$ & $6(3-12)$ & $6(3-12)$ & $5(3-9)$ & $5(3-11)$ & $5(3-10)$ \\
\hline Mean (SD) & $9(8)$ & $9(9)$ & $9(9)$ & $9(12)$ & $9(8)$ & $9(10)$ & $8(9)$ & $7(8)$ & $7(8)$ \\
\hline Range & $1-61$ & $1-50$ & $1-61$ & $1-91$ & $1-48$ & $1-91$ & $1-59$ & $1-59$ & $1-59$ \\
\hline $\begin{array}{l}\text { IMV among all } \\
\text { admissions, } n(\%)^{\mathrm{b}}\end{array}$ & $58(24)^{9}$ & $40(20)$ & $98(22)$ & $53(20)^{9}$ & $59(21)$ & $112(20)$ & $23(13)$ & $32(17)$ & $55(15)$ \\
\hline
\end{tabular}

Abbreviations: ICU, intensive care unit; IMV, invasive mechanical ventilation; IQR, interquartile range; LOS, length of stay; RSV, respiratory syncytial virus; SD, standard deviation; wGA, weeks' gestational age.

aStatistical comparisons not available for combined seasons.

${ }^{\mathrm{b}}$ Among the 702 infants identified during the 2014 to 2015 season, hospital LOS data were available for 678 infants; data pertaining to ICU admission status and need for IMV were available for 684 infants. During the 2015 to 2016 season, 676 infants were identified and hospital LOS data were available for 674 infants; data pertaining to ICU admission status were available for 676 infants, whereas data pertaining to need for IMV were available for 675 infants.

${ }^{c}$ For 2014 to $2015, p=0.001$ for 29 to 32 versus 35 wGA; $p<0.05$ for 33 to 34 versus 35 wGA. For 2015 to $2016, p<0.05$ for 29 to 32 versus 35 wGA (Wilcoxon's rank-sum test).

${ }^{d}$ For 2014 to $2015, p<0.001$ for 29 to 32 versus 35 wGA; $p<0.05$ for 33 to 34 versus 35 wGA. For 2015 to 2016 , differences among the GA groups in the all-identified population were not statistically significant (Pearson's chi-square test).

e Among the 288 infants admitted to the ICU during the 2014 to 2015 season, ICU LOS data were available for 284 infants. During the 2015 to 2016 season, 322 infants were admitted to the ICU, and ICU LOS data were available for 321 infants.

fDifferences among the GA groups in the all-identified population were not statistically significant (Wilcoxon's rank-sum test).

${ }^{9}$ For 2014 to 2015, $p<0.01$ for 29 to 32 versus 35 wGA. For 2015 to 2016, differences among the GA groups in the all-identified population were not statistically significant (Pearson's chi-square test).

associated with high rates of ICU admission and need for IMV (-Fig. 3). Two infants with community-acquired RSV died: one (29 wGA) in 2014 to 2015 and one (30 wGA) in 2015 to 2016. Variables used in univariate analyses and their $p$-values are shown in - Supplementary Table S1 (available in the online version). Multivariate logistic regression analyses confirmed that earlier gestational age and younger chronological age were independently associated with ICU admission and need for IMV (-Table 3 ).

Distribution of RSVH by birth month and gestational age group is presented in -Supplementary Fig. S2A and B (available in the online version). Among all eligible infants ( $N=1,378), 70 \%$ of RSVH occurred among infants born during the months of August through January.

\section{Enrolled Infants}

Of the 1,378 eligible preterm infants with communityacquired RSVH, 481 infants were enrolled for in-depth characterization of their hospitalizations. Of these infants, $44 \%$ were 29 to $32 \mathrm{wGA}, 34 \%$ were 33 to $34 \mathrm{wGA}$, and $22 \%$ were $35 \mathrm{wGA}$. Sociodemographic characteristics and hospital outcomes were similar among those enrolled and all eligible infants ( - Supplementary Tables \$2 and \$3 [available in the online version]).
Of the 481 total infants enrolled over the two seasons, 470 (98\%) had a birth hospitalization discharge date available. Distribution of RSVH based on the month the infants left the birth hospital is presented in - Supplementary Fig. S3A and B (available in the online version). Among infants with RSVH, $73 \%$ were discharged from their birth hospitalization during the months of September through February. Among those enrolled infants discharged from their birth hospitalization from November 1 through March $31(n=267), 46 \%$ of RSVH occurred within the first 30 days after being discharged from their birth hospitalization, and $82 \%$ occurred within 60 days.

Hospital charges obtained from the billing records of all enrolled infants by gestational and chronological age groups are shown in -Supplementary Table 54 (available in the online version). Both chronological age and gestational age affected RSVH charges ( - Fig. 4). Charges were highest among infants aged $<3$ months. Infants born at 29 to 32 wGA who were hospitalized when aged $<3$ months accrued median charges of $\$ 77,209$ (range: $\$ 3,408-\$ 302,721$; interquartile range $[\mathrm{IQR}]=\$ 125,125)$ during 2014 to 2015 $(n=40)$ and $\$ 74,030$ (range: $\$ 6,174-\$ 1,323,095$; IQR $=\$ 123,850)$ during 2015 to $2016(n=42)$. The mean hospital charge for both seasons combined for infants in this chronological age and gestational age group was $\$ 122,301$. 


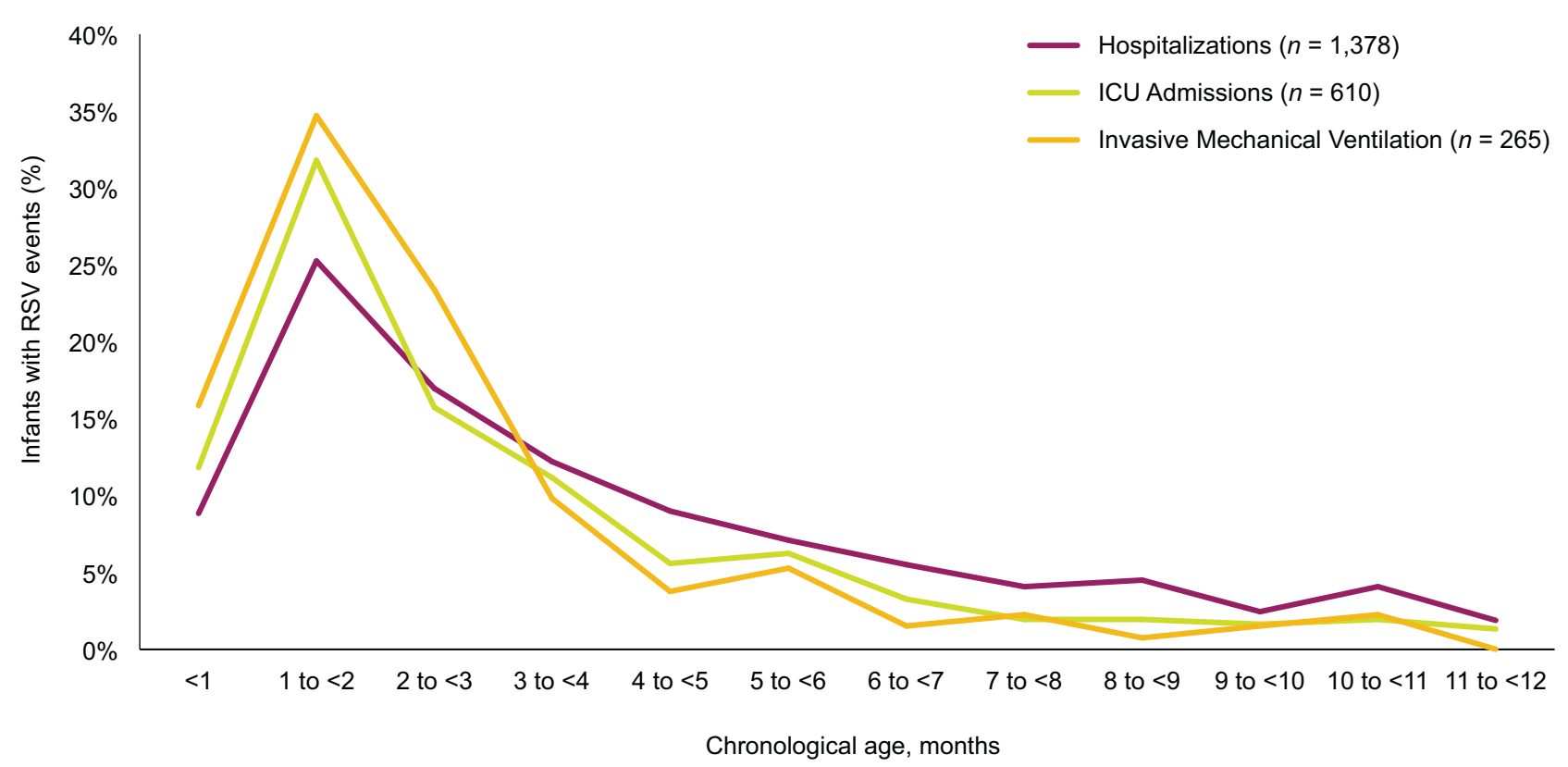

Fig. 2 Chronological age distribution of infants with community-acquired RSV hospitalizations, ICU admissions, and need for IMV for combined seasons (2014-2015 and 2015-2016). ICU, intensive care unit; IMV, invasive mechanical ventilation; RSV, respiratory syncytial virus.

\section{Study Site Standards of Care for RSV Disease Prevention and for RSV Testing}

Study sites were surveyed about site-specific standard-of-care approaches to RSV IP use for otherwise healthy preterm infants 29 to 35 wGA. Approaches varied substantially across the 43 study sites in the 2014 to 2015 RSV season. Some sites followed the 2014 COID guidance, while others adhered more closely to guidelines issued by the National Perinatal Association. ${ }^{22}$ During 2014 to 2015, 40, 21, and 12\% of sites reported routine RSV IP use for infants born at 29 to 31 wGA, 32 to 34 wGA, and 35 wGA, respectively. By 2015 to 2016, 18, 11, and $3 \%$ of the 42 sites reported routine RSV IP use for infants born at 29 to 31 wGA, 32 to 34 wGA, and 35 wGA, respectively, suggesting increased adherence to the 2014 COID guidance. Site-specific standard-of-care approaches to diagnostic testing for RSV were also collected. Twenty-seven (63\%) of the 43 participating sites in the 2014 to 2015 season and 26 (67\%) of the 39 sites responding to the survey in the 2015 to 2016 season reported that diagnostic testing for RSV was routine for all preterm infants hospitalized with a lower respiratory tract illness.

\section{Discussion}

The two-season SENTINEL1 study is the largest study ever conducted of preterm infants requiring hospitalization for laboratory-confirmed RSV infection, and it provides a robust, contemporary, U.S.-specific estimate of the morbidity and costs associated with RSVH among infants born at 29 to 35 wGA. Our results demonstrate substantial ICU admission rates and the need for IMV among preterm infants hospitalized due to RSV who were not receiving RSV IP, particularly during their first 6 months of life. Despite remarkable advances in the care of preterm infants over the past two decades, the results of our study indicate preterm infants have an underlying vulnerability to severe RSV disease that has not changed. $4,7,8$
Additionally, these data demonstrate the substantial direct medical costs of RSVH and the short interval between birth hospitalization discharge and subsequent RSVH. Importantly, among infants discharged from their birth hospitalization during the RSV season, nearly half of subsequent RSVH occur within 30 days of discharge and $82 \%$ within 60 days. Finally, utilizing month of birth hospitalization discharge can enhance risk stratification for prevention of RSVH, which in turn can help reduce the morbidity and subsequent costs associated with RSVH.

A strength of our study is that our results can be generalizable to the United States for the following reasons: (1) our study involved 46 separate U.S. hospitals, including some of the largest children's hospitals in the country; (2) results observed and reported for the 2014 to 2015 and 2015 to 2016 RSV seasons were similar, supporting the generalizability of the study findings across RSV seasons; and (3) a comparison of the detailed data from the enrolled subjects compared with the eligible subjects revealed that these subjects were comparable, supporting generalizability.

As demonstrated from decades of available epidemiologic study reports, earlier gestational age and younger chronological age are associated with a higher risk of serious RSV disease. ${ }^{4,6-8,24-28}$ This underscores the significant impact of chronological age on risk of RSVH and stresses the importance of preventing RSV infection in infants discharged from their birth hospitalization immediately prior to or during the RSV season.

Studies of preterm infants 32 to 35 wGA not receiving RSV IP demonstrated that these infants had two to three times the risk of RSVH compared with full-term infants of the same chronological age, with the risk of RSVH being highest during the first 6 months of life. ${ }^{21-23}$ Historically, the COID guidance recognized that preterm infants 29 to $35 \mathrm{wGA}$ were at high enough risk to warrant RSV IP in certain circumstances that varied among iterations of the guidance. The most recent 


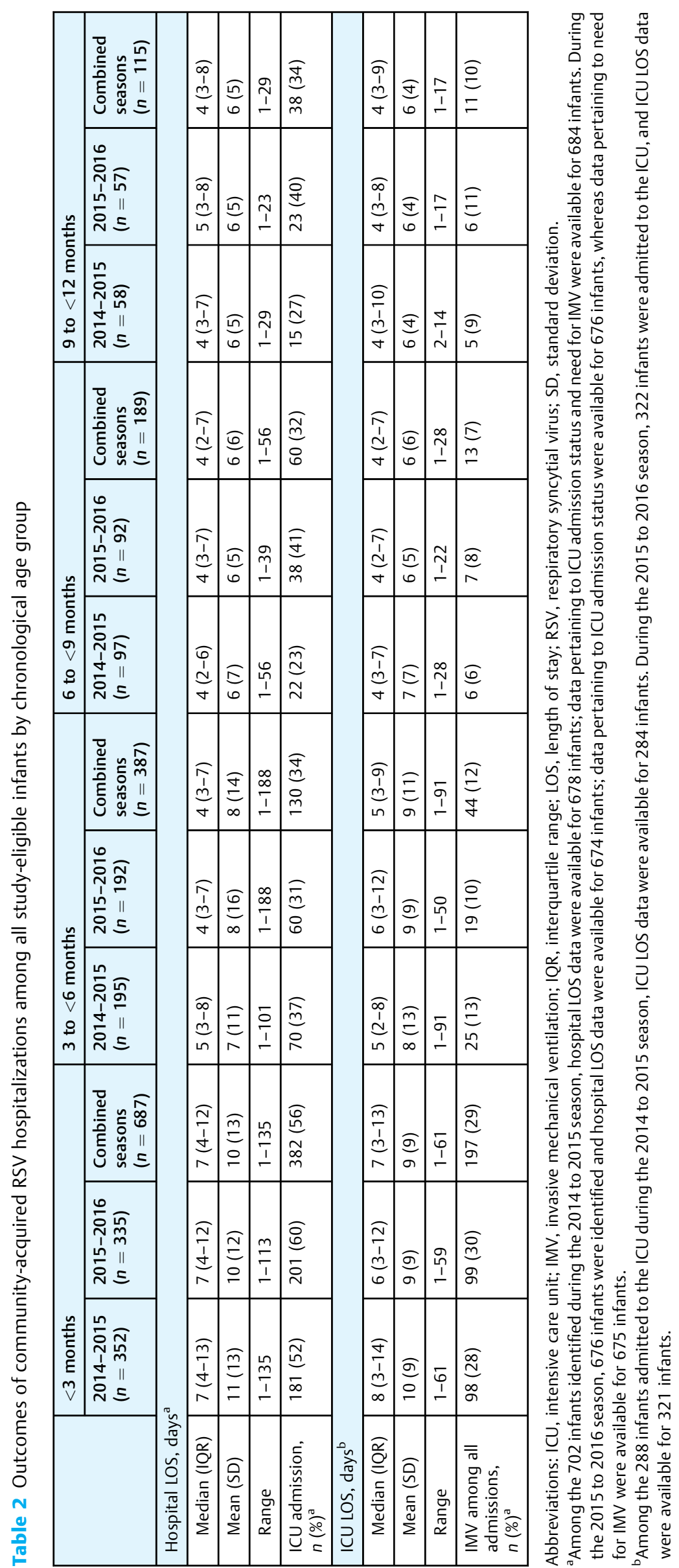




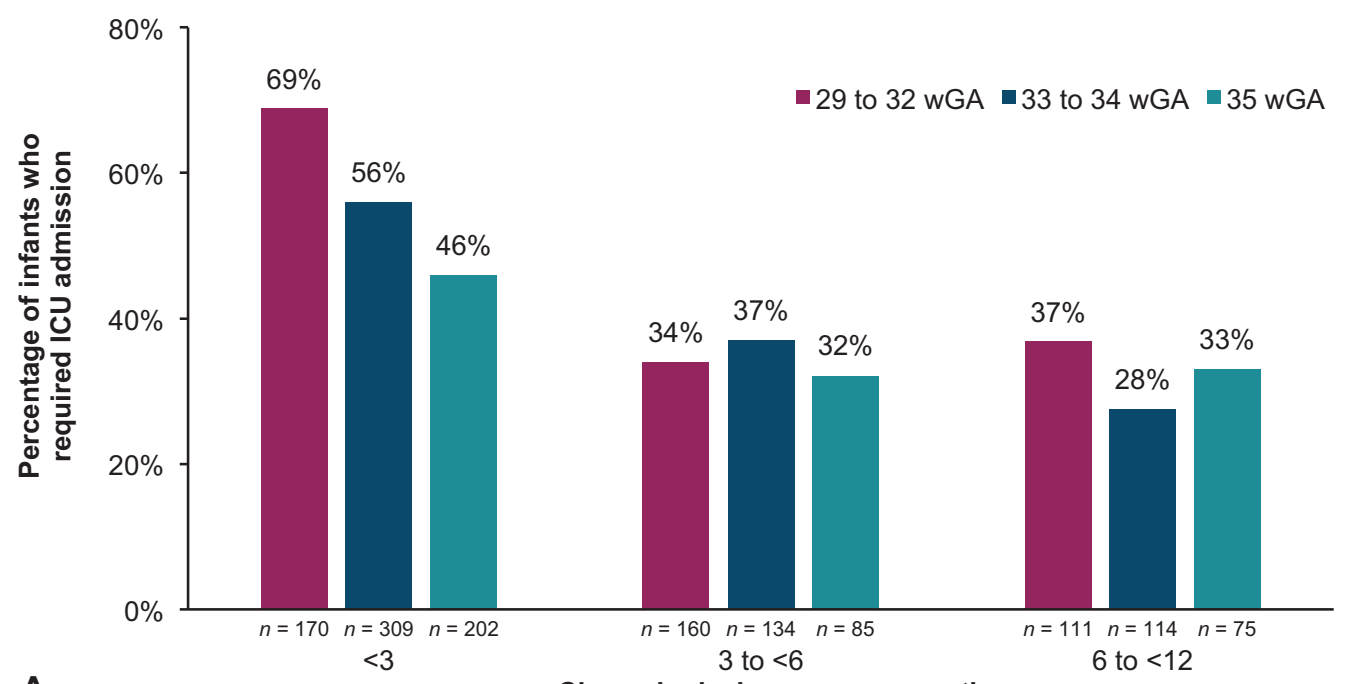

A

Chronological age group, months

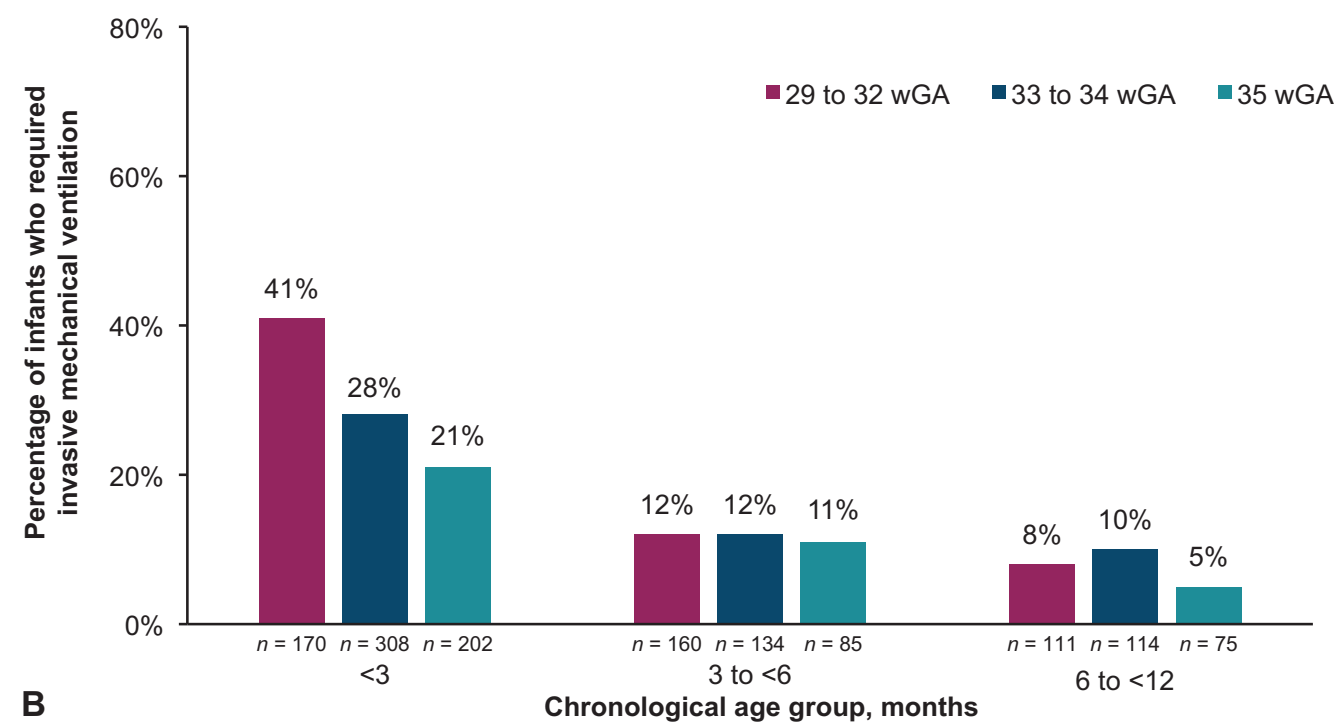

Fig. 3 (A) Percentage of infants born at 29 to 35 wGA hospitalized with community-acquired RSV infection who required ICU admission by gestational age group and chronological age group for combined seasons. (B) Percentage of infants born at 29 to 35 wGA hospitalized with community-acquired RSV infection who required IMV by gestational age group and chronological age group for combined seasons. Among 1,378 infants who had community-acquired RSV disease, data pertaining to ICU admission and need for IMV were available for 1,360 and 1,359 infants, respectively. ICU, intensive care unit; IMV, invasive mechanical ventilation; RSV, respiratory syncytial virus; wGA, weeks' gestational age.

COID guidance statement, released in 2014, was the first to not recommend RSV IP for infants who were born at 29 to 35 wGA without comorbidities.

Although we have shown that infants with greater degrees of prematurity and younger chronological ages suffer significantly greater RSV disease severity once hospitalized compared with infants 35 wGA, a limitation of the SENTINEL1 study is that it does not have a population denominator and therefore cannot evaluate the absolute incidence of RSVH. However, other recent studies have provided population-based data regarding the RSVH incidence and risk relative to term infants in U.S. preterm infants born at 29 wGA and later. Separate reports from data collected from 1999 to 2004 and from 2005 to 2011 showed the risk of RSVH was two- to threefold higher among infants born at 32 to 34 wGA when compared with full-term infants. ${ }^{25,26}$ A third report from a prospective study with laboratory testing for
RSV, which included U.S. infants born at 32 to 35 wGA who were aged $<6$ months and not receiving RSV IP, demonstrated a hospitalization rate of 5 per 100 infant-seasons for RSV disease. Among those infants with the additional risk factors of daycare attendance or preschool-aged siblings, the incidence of RSVH increased to 9 per 100 infant-seasons. ${ }^{24}$ In each of the three studies described, the risk of RSVH was highest during the first 6 months of life, consistent with the SENTINEL1 results presented herein. Additionally, three recent studies demonstrate the rate of $R S V H$ in infants 29 to 34 wGA compared with term infants has increased since the release of the 2014 COID guidance. ${ }^{27,29,30}$

Another limitation of this study is that although hospital charges are reported, actual reimbursement amounts may differ. Hospital charges reported here do not include physician fees and also do not account for reimbursements that are commonly negotiated between hospitals and insurance carriers. 
Table 3 Multivariate logistic regression to evaluate the independent associations of gestational age group and chronological age group on requirements for ICU admission and IMV for combined seasons

\begin{tabular}{|c|c|c|c|c|}
\hline & \multicolumn{2}{|l|}{ ICU admission } & \multicolumn{2}{|l|}{ Need for IMV } \\
\hline & Odds ratio $(95 \% \mathrm{Cl})$ & $p$-Value & Odds ratio $(95 \% \mathrm{Cl})$ & $p$-Value \\
\hline \multicolumn{5}{|c|}{ By gestational age group } \\
\hline $29-32$ wGA & $1.76(1.30,2.37)$ & $<0.001$ & $2.08(1.42,3.06)$ & $<0.001$ \\
\hline $33-34$ wGA & $1.27(0.96,1.68)$ & 0.09 & $1.43(1.00,2.06)$ & 0.05 \\
\hline 35 wGA & Reference level & - & Reference level & - \\
\hline \multicolumn{5}{|c|}{ By chronological age group } \\
\hline$<3 \mathrm{mo}$ & $2.72(1.77,4.16)$ & $<0.0001$ & $4.20(2.19,8.06)$ & $<0.0001$ \\
\hline 3 to $<6 \mathrm{mo}$ & $1.00(0.64,1.57)$ & 0.99 & $1.21(0.60,2.45)$ & 0.59 \\
\hline 6 to $<9 \mathrm{mo}$ & $0.93(0.56,1.54)$ & 0.78 & $0.71(0.30,1.64)$ & 0.41 \\
\hline 9 to $<12 \mathrm{mo}$ & Reference level & - & Reference level & - \\
\hline
\end{tabular}

Abbreviations: $\mathrm{Cl}$, confidence interval; ICU, intensive care unit; IMV, invasive mechanical ventilation; wGA, weeks' gestational age.

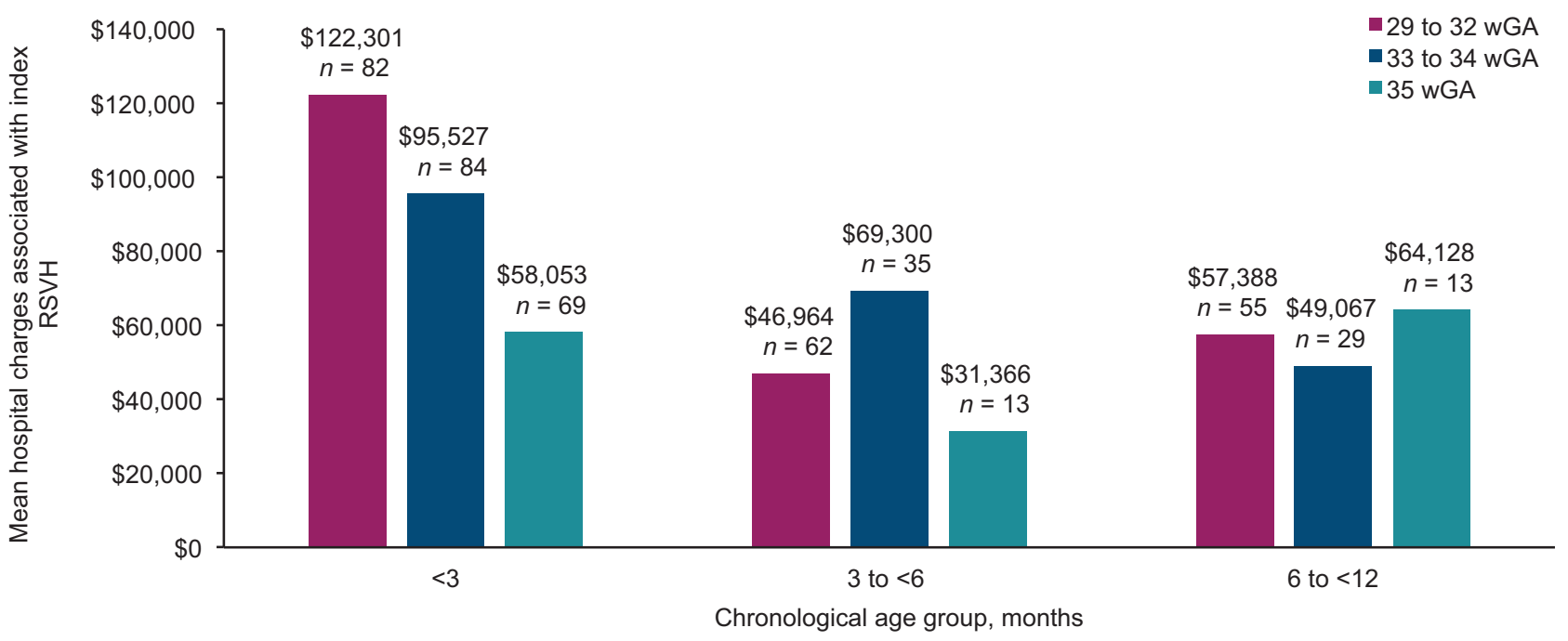

Fig. 4 Mean hospital charges for infants born at 29 to 35 wGA hospitalized with community-acquired RSV infection by gestational age group and chronological age group for combined seasons. RSV, respiratory syncytial virus; RSVH, respiratory syncytial virus hospitalization; wGA, weeks' gestational age.

\section{Conclusion}

Despite recent advances in neonatal care, RSV disease continues to cause significant morbidity in U.S. preterm infants born at 29 to 35 wGA. Hospitalization for RSV infection frequently involves intensive care. A substantial portion of infants who need intensive care requires invasive ventilatory support, further contributing to the high hospital charges associated with this illness. Until new methods of prevention are available, RSV IP with palivizumab can reduce the significant burden of RSV disease in infants 29 to 35 wGA.

\section{Note}

Portions of this work were presented at IDWeek 2016, New Orleans, LA, October 26-30, 2016; the Society of Critical Care Medicine's 46th Critical Care Congress, Honolulu, Hawaii, January 21-25, 2017; the Pediatric Academic Societies (PAS) 2017 Meeting, San Francisco, CA,
May 6-9, 2017; IDWeek 2017, San Diego, CA, October 4-8, 2017; and the PAS 2018 Meeting, Toronto, ON, Canada, May 5-8, 2018.

\section{Funding}

This study was sponsored by AstraZeneca. Editorial support was provided by The Lockwood Group, which was in accordance with Good Publication Practice (GPP3) guidelines and funded by AstraZeneca.

\section{Conflict of Interest}

E.J.A., J.P.D., E.A.F.S., L.R.K., M.L.F., P.S.P., C.M.E., R.C.W., L.I. W., R.Y., P.A.C., J.B.D., and N.H. are independent investigators who have received research support from AstraZeneca/MedImmune. J.P.D., E.A.F.S., L.R.K., and M.L.F. also received travel support from AstraZeneca/MedImmune to present the results of this or other research studies at scientific meetings. J.P.D., M.L.F., and P.S.P. have served on 
the speakers' bureau for AstraZeneca/MedImmune. E.J.A., E.A.F.S., and J.P.D. have served as consultants to AbbVie; P.A.C. has received research support from AbbVie. S.J.M. is an independent statistical consultant to AstraZeneca. K.K.M. and C.S.A. are employees of AstraZeneca and hold stock. V.R.K. and C.P.R. were employees of AstraZeneca at the time this research was conducted. C.P.R. is a current employee of Sobi Inc.

\section{References}

1 Leader S, Kohlhase K. Recent trends in severe respiratory syncytial virus (RSV) among US infants, 1997 to 2000. J Pediatr 2003;143(5, Suppl):S127-S132

2 Mandell GL, Bennett JE, Dolin R. Mandell, Douglas and Bennett's Principles and Practice of Infectious Diseases, 4th ed. New York: Churchill Livingstone; 1995

3 Jain S, Williams DJ, Arnold SR, et al; CDC EPIC Study Team. Community-acquired pneumonia requiring hospitalization among U.S. children. N Engl J Med 2015;372(09):835-845

4 Boyce TG, Mellen BG, Mitchel EF Jr, Wright PF, Griffin MR. Rates of hospitalization for respiratory syncytial virus infection among children in Medicaid. J Pediatr 2000;137(06):865-870

5 Langley GF, Anderson LJ. Epidemiology and prevention of respiratory syncytial virus infections among infants and young children. Pediatr Infect Dis J 2011;30(06):510-517

6 Stevens TP, Sinkin RA, Hall CB, Maniscalco WM, McConnochie KM. Respiratory syncytial virus and premature infants born at 32 weeks' gestation or earlier: hospitalization and economic implications of prophylaxis. Arch Pediatr Adolesc Med 2000;154(01):55-61

7 Horn SD, Smout RJ. Effect of prematurity on respiratory syncytial virus hospital resource use and outcomes. J Pediatr 2003;143(5, Suppl):S133-S141

8 Law BJ, Langley JM, Allen U, et al. The Pediatric Investigators Collaborative Network on Infections in Canada study of predictors of hospitalization for respiratory syncytial virus infection for infants born at 33 through 35 completed weeks of gestation. Pediatr Infect Dis J 2004;23(09):806-814

9 DeVincenzo JP, McClure MW, Symons JA, et al. Activity of oral ALS008176 in a respiratory syncytial virus challenge study. $\mathrm{N}$ Engl J Med 2015;373(21):2048-2058

10 DeVincenzo JP, Whitley RJ, Mackman RL, et al. Oral GS-5806 activity in a respiratory syncytial virus challenge study. $\mathrm{N}$ Engl J Med 2014;371(08):711-722

11 Simões EA, DeVincenzo JP, Boeckh M, et al. Challenges and opportunities in developing respiratory syncytial virus therapeutics. J Infect Dis 2015;211(Suppl 1):S1-S20

12 IMpact RSV-Study Group. Palivizumab, a humanized respiratory syncytial virus monoclonal antibody, reduces hospitalization from respiratory syncytial virus infection in high-risk infants. Pediatrics 1998;102:531-537

13 Blanken MO, Rovers MM, Molenaar JM, et al; Dutch RSV Neonatal Network. Respiratory syncytial virus and recurrent wheeze in healthy preterm infants. N Engl J Med 2013;368(19):1791-1799

14 Anderson EJ, Carosone-Link P, Yogev R, Yi J, Simões EAF. Effectiveness of palivizumab in high-risk Infants and children: a propensity score weighted regression analysis. Pediatr Infect Dis J 2017;36(08):699-704

15 American Academy of Pediatrics Committee on Infectious Diseases and Committee of Fetus and Newborn. Prevention of respiratory syncytial virus infections: indications for the use of palivizumab and update on the use of RSV-IGIV. Pediatrics 1998; 102(05):1211-1216
16 American Academy of Pediatrics Committee on Infectious Diseases and Committee on Fetus and Newborn. Revised indications for the use of palivizumab and respiratory syncytial virus immune globulin intravenous for the prevention of respiratory syncytial virus infections. Pediatrics 2003;112(6 Pt 1): 1442-1446

17 American Academy of Pediatrics Subcommittee on Diagnosis and Management of Bronchiolitis. Diagnosis and management of bronchiolitis. Pediatrics 2006;118(04):1774-1793

18 Committee on Infectious Diseases. From the American Academy of Pediatrics: policy statements-modified recommendations for use of palivizumab for prevention of respiratory syncytial virus infections. Pediatrics 2009;124(06):1694-1701

19 American Academy of Pediatrics. Respiratory syncytial virus. In: Pickering LK, Baker CJ, Kimberlin DW, Long SS, eds. Red Book: 2012 Report of the Committee on Infectious Diseases, 29th ed. Elk Grove Village, IL: American Academy of Pediatrics; 2012: 609-618

20 American Academy of Pediatrics Committee on Infectious Diseases; American Academy of Pediatrics Bronchiolitis Guidelines Committee. Updated guidance for palivizumab prophylaxis among infants and young children at increased risk of hospitalization for respiratory syncytial virus infection. Pediatrics 2014; 134(02):415-420

21 Boyce TG. More on viral bronchiolitis in children. N Engl J Med 2016;375(12):1199-1200

22 Goldstein M, Merritt TA, Phillips R, et al. National Perinatal Association 2015 Respiratory Syncytial Virus (RSV) Prevention Guideline. Neonatology Today [serial online]. 2014;9:1-11. Available at: https:// www.neonatologytoday.net/issues.php\#2014issues. Accessed August 15, 2018

23 Anderson EJ, Krilov LR, DeVincenzo JP, et al. SENTINEL1: an observational study of respiratory syncytial virus hospitalizations among US infants born at 29 to 35 weeks' gestational age not receiving immunoprophylaxis. Am J Perinatol 2017;34(01): 51-61

24 Ambrose CS, Anderson EJ, Simões EA, et al. Respiratory syncytial virus disease in preterm infants in the U.S. born at 32-35 weeks gestation not receiving immunoprophylaxis. Pediatr Infect Dis J 2014;33(06):576-582

25 Helfrich AM, Nylund CM, Eberly MD, Eide MB, Stagliano DR. Healthy late-preterm infants born $33-36+6$ weeks gestational age have higher risk for respiratory syncytial virus hospitalization. Early Hum Dev 2015;91(09):541-546

26 Winterstein AG, Knox CA, Kubilis P, Hampp C. Appropriateness of age thresholds for respiratory syncytial virus immunoprophylaxis in moderate-preterm infants: a cohort study. JAMA Pediatr 2013; 167(12):1118-1124

27 Rajah B, Sánchez PJ, Garcia-Maurino C, Leber A, Ramilo O, Mejias A. Impact of the updated guidance for palivizumab prophylaxis against respiratory syncytial virus infection: a single center experience. J Pediatr 2017;181:183-188

28 Blanken MO, Paes B, Anderson EJ, et al. Risk scoring tool to predict respiratory syncytial virus hospitalisation in premature infants. Pediatr Pulmonol 2018;53(05):605-612

29 Kong AM, Krilov LR, Fergie J, et al. The 2014-2015 national impact of the 2014 American Academy of Pediatrics guidance for respiratory syncytial virus immunoprophylaxis on preterm infants born in the United States. Am J Perinatol 2018;35(02): 192-200

30 Goldstein M, Krilov LR, Fergie J, et al. Respiratory syncytial virus hospitalizations among US preterm infants compared with term infants before and after the 2014 American Academy of Pediatrics guidance on immunoprophylaxis: 2012-2016. Am J Perinatol 2018;35(14):1433-1442 